\title{
CONVERGENCE OF MULTIVARIATE POLYNOMIALS INTERPOLATING ON A TRIANGULAR ARRAY \\ BY
}

\author{
T. N. T. GOODMAN AND A. SHARMA
}

\begin{abstract}
Given a triangular array of complex numbers, it is well known that for any function $f$ smooth enough, there is a unique polynomial $G_{n} f$ of degree $\leq n$ such that on each of the first $n+1$ rows of the array the divided difference of $G_{n} f$ coincides with that of $f$. This result has recently been generalized to give a unique polynomial $\mathcal{G}_{n} f$ in $k$ variables $(k>1)$ of total degree $\leq n$ which interpolates a given function $f$ on a triangular array in $C^{k}$. In this paper we extend some results of A. O. Gelfond and derive formulas for $\mathcal{G}_{n} f$ and $f-\mathcal{G}_{n} f$ to prove some results on convergence of $\mathcal{G}_{n} f$ to $f$ as $n \rightarrow \infty$ under various conditions on $f$ and on the triangular array.
\end{abstract}

1. Introduction. Suppose we have a triangular array of points in $\mathbf{R}^{k}, k \geq 1$, whose $n$th row $\rho_{n}$ is given by

$$
\rho_{n}:=\left\{x^{0 n}, \ldots, x^{n n}\right\}, \quad n=0,1, \ldots
$$

First suppose $k=1$ and let $\left[\rho_{n}\right] f$ denote the divided difference of a function $f$ with respect to the points of the row $\rho_{n}$. For $k=1$, Gelfond [3, p. 39] showed that for any integer $n \geq 0$ and any given function $f$ for which the divided differences have a meaning, there is a unique polynomial of degree $\leq n$ satisfying

$$
\left[\rho_{j}\right] G_{n} f=\left[\rho_{j}\right] f, \quad j=0,1, \ldots, n .
$$

If for $j=0,1, \ldots, n$ the $j$ th column comprises only one element $x^{j}$, i.e., $x^{j}=x^{j j}=$ $\cdots=x^{j n}$, then $G_{n} f$ reduces to the polynomial interpolating $f$ at $x^{0}, \ldots, x^{n}$. If instead each row comprises only one element, i.e., $x^{j}=x^{0 j}=\cdots=x^{j j}, j=$ $0,1, \ldots, n$, then the interpolation procedure is known as Abel-Gontcharoff interpolation.

In $[2, \S 5]$ Gelfond's interpolation procedure is generalized to $k>1$. To describe this, we must first make some definitions. For $\rho=\left\{x^{0}, \ldots, x^{n}\right\} \subset \mathbf{R}^{k}$ and $f \in$ $C\left(\mathbf{R}^{k}\right)$, we define

$$
\int_{[\rho]} f(x) d x=\int_{\left[x^{0}, \ldots, x^{n}\right]} f(x) d x=\int_{S^{n}} f\left(\nu_{0} x^{0}+\cdots+\nu_{n} x^{n}\right) d \nu_{1} \cdots d \nu_{n},
$$

where $S^{n}=\left\{\left(\nu_{1}, \ldots, \nu_{n}\right): \nu_{j} \geq 0, j=1, \ldots, n ; \sum_{1}^{n} \nu_{j} \leq 1\right\}$ and $\sum_{0}^{n} \nu_{j}=1$.

The term $d x$ in (1.3) will play an important role in distinguishing with respect to which variable the integral is being taken. If this is unnecessary, we revert to the usual notation (introduced in [5]) and write

$$
\int_{[\rho]} f(x) d x=\int_{[\rho]} f .
$$

Received by the editors December 13, 1982 and, in revised form, October 3, 1983.

1980 Mathematics Subject Classification. Primary 32A05, 30E05.

Key words and phrases. Kergin interpolation, multivariate approximation, divided difference. 
It is shown in [2] that for any $n \geq 0$ and any $f \in C^{n}\left(\mathbf{R}^{k}\right)$, there is a unique polynomial $\mathcal{G}_{n}\left(f \mid \rho_{0}, \ldots, \rho_{n}\right)$ (or $\mathcal{G}_{n} f$ ) of total degree $\leq n$ satisfying

$$
\int_{\left[\rho_{j}\right]} D^{\alpha} \mathcal{G}_{n} f=\int_{\left[\rho_{j}\right]} D^{\alpha} f, \quad \forall \alpha \text { with }|\alpha|=j(j=0,1, \ldots, n),
$$

where $\alpha=\left(\alpha_{1}, \ldots, \alpha_{k}\right)$,

$$
|\alpha|=\alpha_{1}+\cdots+\alpha_{k} \text { and } D^{\alpha}=\partial^{\alpha_{1}} / \partial x_{1}^{\alpha_{1}} \cdots \partial^{\alpha_{k}} / \partial x_{k}^{\alpha_{k}} .
$$

Using the Hermite-Genocchi formula (e.g., [5])

$$
\left[t_{0}, \ldots, t_{n}\right] f=\int_{\left[t_{0}, \ldots, t_{n}\right]} f^{(n)}
$$

we see that $\mathcal{G}_{n} f$ reduces to $G_{n} f$ when $k=1$.

If $k>1$ and if for $j=0,1, \ldots, n$ the $j$ th column of the triangular array comprises only one element, then the above reduces to Kergin interpolation at $x^{0}, \ldots, x^{n}$, a form of multivariate interpolation recently introduced in [4]. Further if we have that $x^{0}=\cdots=x^{n}$, then $\mathcal{G}_{n} f$ reduces to the Taylor polynomial of $f$ at $x^{0}$. A convenient remainder formula for Kergin interpolation is given in [5]. In [2] the authors remark that it would be interesting to have an analogous remainder formula for $f-\mathcal{G}_{n} f$ when $\mathcal{G}_{n} f$ satisfies (1.4). Such a formula is derived in $\S 2$ together with a corresponding formula for $\mathcal{G}_{n} f$.

The above definitions extend in an obvious way to a triangular array in $\mathbf{C}^{k}$ and a holomorphic function $f$. For such a case with $k=1$, Gelfond proves several results about convergence of $G_{n} f$ to $f$ as $n \rightarrow \infty$, under various conditions on $f$ and the triangular array $[3$, pp. $36-54]$. In $\S 5$ we state and prove some generalizations of these results to $k>1$. Our proofs generalize Gelfond's proofs step by step and are based on some lemmas in $\S 3$ and on some estimates in $\S 4$.

Recently T. Bloom [1] has generalized another result of Gelfond on the convergence of Lagrange interpolation polynomials to the corresponding case of Kergin interpolation. He also remarks that "it is quite likely that other results on Lagrange interpolation for functions of one complex variable can be generalized to functions of several complex variables by considering Kergin interpolation".

2. Basic formulas. We first state some properties of the integral (1.3). The proofs follow easily from the definition and are omitted. For any set $S,[S]$ will denote its closed convex hull.

Proposition 1. Suppose $T: \mathbf{R}^{k} \rightarrow \mathbf{R}^{l}$ is an affine map, i.e., $T x=A x+c$ where $A: \mathbf{R}^{k} \rightarrow \mathbf{R}^{l}$ is linear and $c \in \mathbf{R}^{l}$. Then for any $x^{0}, x^{1}, \ldots, x^{n}$ in $\mathbf{R}^{k}$ and $f \in C\left[\left\{T x^{0}, \ldots, T x^{n}\right\}\right]$, we have

$$
\int_{\left[T x^{0}, \ldots, T x^{n}\right]} f(y) d y=\int_{\left[x^{0}, \ldots, x^{n}\right]} f(T x) d x .
$$

Proposition 2. For $x^{0}, \ldots, x^{n}$ in $\mathbf{R}^{k}$ and $f \in C\left[\left\{x^{0}, \ldots, x^{n}\right\}\right]$

$$
\int_{\left[x^{0}, \ldots, x^{n}\right]} f(x) d x \leq \int_{\left[x^{0}, \ldots, x^{n}\right]}|f(x)| d x .
$$


Proposition 3. For $a, b \in \mathbf{R}^{k}$ and $f \in C^{1}[\{a, b\}]$

$$
\int_{[a, b]} D_{b-a} f=f(b)-f(a)=\int_{[0, b]} D_{b} f-\int_{[0, a]} D_{a} f .
$$

Here we have used the notation that for any $y \in \mathbf{R}^{k}, D_{y}$ denotes the directional derivative

$$
D_{y}:=\sum_{j=1}^{k} y_{j} D_{j}, \quad D_{j}=\frac{\partial}{\partial x_{j}}, \quad y=\left(y_{1}, \ldots, y_{k}\right) .
$$

The following theorem is proved in $[2]$. Since there are some minor errors in presentation, we reproduce it here in a revised form.

TheOREM A (CAVARETTA, MiCChelli AND ShARMA). There is a unique polynomial $\mathcal{G}_{n}\left(f \mid \rho_{0}, \ldots, \rho_{n}\right)(x)$ of degree $\leq n$ satisfying equation (1.4) for $f(x) \in$ $C^{n}\left[\rho_{0} \cup \cdots \cup \rho_{n}\right]$, where $\rho_{0}, \ldots, \rho_{n}$ are given by $(1.1)$.

PROOF. Consider first the case $k=1$ with a triangular array of rows $\tau_{n}=$ $\left\{t_{0 n}, \ldots, t_{n n}\right\}, n=0,1, \ldots$, where $t_{0 n}, \ldots, t_{n n}$ where $t_{0 n}, \ldots, t_{n n}$ are real numbers. In this case we can construct [3, p. 37] the fundamental polynomials of interpolation $p_{n}(t)=p_{n}\left(t \mid \tau_{0}, \ldots, \tau_{n}\right), n=0,1, \ldots$, satisfying

$$
\left[\tau_{j}\right] p_{n}=\delta_{j n}, \quad j=0,1, \ldots
$$

Let $\{t\} \cup \tau_{0} \cup \cdots \cup \tau_{n-1}=\left\{t_{1}, \ldots, t_{N}\right\}$. Then $p_{n}\left(t \mid \tau_{0}, \ldots, \tau_{n-1}\right)$ is a homogeneous polynomial of degree $n$ in $t_{1}, \ldots, t_{N}$ and so can be written

$$
p_{n}\left(t \mid \tau_{0}, \ldots, \tau_{n-1}\right)=\sum_{|\alpha|=n} a_{\alpha} t_{1}^{\alpha_{1}} \cdots t_{N}^{\alpha_{N}}
$$

We now take our triangular array in $\mathbf{R}^{k}$ and define correspondingly

$$
\{x\} \cup \rho_{0} \cup \cdots \cup \rho_{n-1}=\left\{x^{1}, \ldots, x^{N}\right\} .
$$

Next we define a differential operator $q_{n}\left(D \mid x ; \rho_{0}, \ldots, \rho_{n-1}\right)$ on $C^{n}\left(\mathbf{R}^{k}\right)$ :

$$
q_{n}\left(D \mid x ; \rho_{0}, \ldots, \rho_{n-1}\right)=\sum_{|\alpha|=n} a_{\alpha} D_{x^{1}}^{\alpha_{1}} \cdots D_{x^{N}}^{\alpha_{N}}
$$

Finally we define

$$
\mathcal{G}_{n}\left(f \mid \rho_{0}, \ldots, \rho_{n}\right)(x)=\sum_{j=0}^{n} \int_{\left[\rho_{j}\right]} q_{j}\left(D \mid x ; \rho_{0}, \ldots, \rho_{j-1}\right) f(y) d y
$$

We note that if

$$
f(y)=g(\lambda \cdot y), \quad \lambda \in \mathbf{R}^{k}, g \in C^{n}\left[\lambda \cdot \rho_{0} \cup \cdots \cup \lambda \cdot \rho_{n}\right],
$$


where $\lambda \cdot y=\lambda_{1} y_{1}+\cdots+\lambda_{k} y_{k}$, then

$$
\begin{aligned}
\mathcal{G}_{n}\left(f \mid \rho_{0}, \ldots, \rho_{n}\right)(x)=\sum_{j=0}^{n} \int_{\left[\rho_{j}\right]} \sum_{|\alpha|=j} a_{\alpha}\left(\lambda \cdot x^{1}\right)^{\alpha_{1}} \cdots\left(\lambda \cdot x^{N}\right)^{\alpha_{N}} g^{(j)}(\lambda \cdot y) d y \\
\quad=\sum_{j=0}^{n} \int_{\left[\lambda \cdot \rho_{j}\right]} g^{(j)}(t) d t \sum_{|\alpha|=j} a_{\alpha}\left(\lambda \cdot x^{1}\right)^{\alpha_{1}} \cdots\left(\lambda \cdot x^{N}\right)^{\alpha_{N}} \quad \text { by }(2.1) \\
=\sum_{j=0}^{n}\left[\lambda \cdot \rho_{j}\right] g \cdot p_{j}\left(\lambda \cdot x \mid \lambda \cdot \rho_{0}, \ldots, \lambda \cdot \rho_{j-1}\right) \quad \text { by }(1.5),(2.5) \\
=G_{n}\left(g \mid \lambda \cdot \rho_{0}, \ldots, \lambda \cdot \rho_{n}\right)(\lambda \cdot x) \quad \text { by }(2.4) .
\end{aligned}
$$

In the terminology of [2], this says that $\mathcal{G}_{n}$ lifts $G_{n}$. It follows easily, as in [2], that (1.4) is satisfied. Since functions of the form (2.8) are dense in $C^{n}\left[\rho_{0} \cup \cdots \cup \rho_{n}\right]$, it follows that (1.4) is satisfied for all $f \in C^{n}\left[\rho_{0} \cup \cdots \cup \rho_{n}\right]$.

Next we show that the polynomial $\mathcal{G}_{n} f$ is the only polynomial of degree $\leq n$ satisfying (1.4). It is enough to show that if $p$ is a polynomial of degree $\leq n$ satisfying

$$
\int_{\left[\rho_{j}\right]} D^{\alpha} p=0, \quad \forall \alpha \text { with }|\alpha|=j, j=0,1, \ldots, n
$$

then $p \equiv 0$. This is easily seen, since for any multi-index $\beta$ with $|\beta|=n$, we have

$$
0=\int_{\left[\rho_{n}\right]} D^{\beta} p=D^{\beta} p \int_{\left[\rho_{n}\right]} d x=D^{\beta} p / n !
$$

Thus $p$ is of degree $\leq n-1$. Continuing this process shows that $p \equiv 0$.

The following result will be useful later.

PROPOSITION 4. Suppose $T: \mathbf{R}^{k} \rightarrow \mathbf{R}^{l}$ is an affine map. For $\rho_{j} \subset \mathbf{R}^{k}, j=$ $0,1, \ldots, n$, and $g \in C^{n}\left[T \rho_{0} \cup \cdots \cup T \rho_{n}\right]$, define

$$
f(x)=g(T x), \quad \mathcal{G}_{n} f=\mathcal{G}_{n}\left(f \mid \rho_{0}, \ldots, \rho_{n}\right), \quad \mathcal{G}_{n} g=\mathcal{G}_{n}\left(g \mid T \rho_{0}, \ldots, T \rho_{n}\right)
$$

Then

$$
\mathcal{G}_{n} f(x)=\mathcal{G}_{n} g(T x)
$$

PROOF. Let $H(x)=\mathcal{G}_{n} g(T x)$. We need to show that

$$
\int_{\left[\rho_{j}\right]} D^{\alpha} \not H=\int_{\left[\rho_{j}\right]} D^{\alpha} f \quad \forall \alpha \text { with }|\alpha|=j, j=0,1, \ldots, n .
$$


Let $(T x)_{i}=\sum_{j=1}^{k} a_{i j} x_{j}+c_{i}, i=1, \ldots, l$. Then

$$
\begin{aligned}
\int_{\left[\rho_{j}\right]} D_{k_{1}} & \cdots D_{k_{j}} H(x) d x=\int_{\left[\rho_{j}\right]} \sum_{i_{1}, \ldots, i_{j}=1}^{l} a_{i_{1} k_{1}} \cdots a_{i_{j} k_{j}} D_{i_{1}} \cdots D_{i_{j}} \mathcal{G}_{n} g(T x) d x \\
& =\sum_{i_{1}, \ldots, i_{j}=1}^{l} a_{i_{1} k_{1}} \cdots a_{i_{j} k_{j}} \int_{\left[T \rho_{j}\right]} D_{i_{1}} \cdots D_{i_{j}} g_{n} g(y) d y, \quad \text { by }(2.1) \\
& =\sum_{i_{1}, \ldots, i_{j}=1}^{l} a_{i_{1} k_{1}} \cdots a_{i_{j} k_{j}} \int_{\left[T \rho_{j}\right]} D_{i_{1}} \cdots D_{i_{j}} g(y) d y \\
& =\int_{\left[\rho_{j}\right]} \sum_{i_{1}, \ldots, i_{j}=1}^{l} a_{i_{1} k_{1}} \cdots a_{i_{j} k_{j}} D_{i_{1}} \cdots D_{i_{j}} g(T x) d x \\
& =\int_{\left[\rho_{j}\right]} D_{k_{1}} \cdots D_{k_{j}} f(x) d x .
\end{aligned}
$$

This completes the proof of $(2.8 \mathrm{a})$.

To simplify the forthcoming formulas we shall put $x^{00}=y^{0}$ and for $j=1,2, \ldots$, we put

$$
\int_{j} f(x) d x=j ! \int_{\left[\rho_{j}\right]} f(x) d x .
$$

Now for $k=1$, there are the following formulas for $f \in C^{n+1}\left[\{t\} \cup \rho_{0} \cup \cdots \cup \rho_{n}\right]$ $[3$, p. 37]:

$$
G_{n} f(t)=\sum_{j=0}^{n}\left[\rho_{j}\right] f \cdot p_{j}(t)
$$

where $p_{0}(t)=1$, and for $j=1, \ldots, n$

$$
p_{j}(t)=j ! \int_{1} \cdots \int_{j-1} \int_{y_{0}}^{t} \int_{y_{1}}^{t_{1}} \cdots \int_{y_{j-1}}^{t_{j-1}} d t_{j} \cdots d t_{1} d y_{j-1} \cdots d y_{1} .
$$

Putting $R_{n} f(t)=f(t)-G_{n} f(t)$, we have

$$
R_{n} f(t)=\int_{1} \int_{2} \cdots \int_{n} \int_{y_{0}}^{t} \int_{y_{1}}^{t_{1}} \cdots \int_{y_{n}}^{t_{n}} f^{(n+1)}\left(t_{n+1}\right) d t_{n+1} \cdots d t_{1} d y_{n} \cdots d y_{1} .
$$

These results are generalized to $k \geq 1$ as follows:

THEOREM 1. For $f \in C^{n+1}\left[\{x\} \cup \rho_{0} \cup \cdots \cup \rho_{n}\right]$, we have the following formulas:

$$
\mathcal{G}_{n} f(x)=\sum_{j=0}^{n} P_{j} f(x)
$$

where $P_{0} f(x)=f\left(y^{0}\right)$, and for $j=1, \ldots, n$ we have

$$
\begin{aligned}
P_{j} f(x)=\int_{1} \cdots \int_{j} \int_{\left[y^{0}, x\right]} \int_{\left[y^{1}, x^{1}\right]} \cdots \int_{\left[y^{j-1}, x^{j-1}\right]} D_{x-y^{0}} \prod_{\nu=1}^{j-1} D_{x^{\nu}-y^{\nu}} \\
\times f\left(y^{j}\right) d x^{j} \cdots d x^{1} d y^{j} \cdots d y^{1}
\end{aligned}
$$


Putting $R_{n} f(x)=f(x)-\mathcal{G}_{n} f(x)$, we have

$$
\begin{aligned}
R_{n} f(x)=\int_{1} \cdots \int_{n} \int_{\left[y^{0}, x\right]} \int_{\left[y^{1}, x^{1}\right]} \cdots & \int_{\left[y^{n}, x^{n}\right]} D_{x-y^{0}} \prod_{\nu=1}^{n} D_{x^{\nu}-y^{\nu}} \\
& \times f\left(x^{n+1}\right) d x^{n+1} \cdots d x^{1} d y^{n} \cdots d y^{1} .
\end{aligned}
$$

ProOF. We first prove (2.13). As in the proof of Theorem A, it is sufficient to prove it for functions of the form $f(x)=g(\lambda \cdot x), \lambda \in \mathbf{R}^{k}$, where

$$
g \in C^{n+1}\left[\{\lambda \cdot x\} \cup \lambda \cdot \rho^{0} \cup \cdots \cup \lambda \cdot \rho^{n}\right] .
$$

In this case the right-hand side of (2.13) becomes

$$
\begin{aligned}
\int_{1} \cdots \int_{n} \int_{\left[y^{0}, x\right]} \cdots \int_{\left[y^{n}, x^{n}\right]}(\lambda \cdot x- & \left.\lambda \cdot y^{0}\right) \prod_{\nu=1}^{n}\left(\lambda \cdot x^{\nu}-\lambda \cdot y^{\nu}\right) g^{(n+1)} \\
& \times\left(\lambda \cdot x^{n+1}\right) d x^{n+1} \cdots d x^{1} \cdot d y^{n} \cdots d y^{1} .
\end{aligned}
$$

Since

$$
\begin{aligned}
& \lambda \cdot\left(x^{n}-y^{n}\right) \int_{\left[y^{n}, x^{n}\right]} g^{(n+1)}\left(\lambda \cdot x^{n+1}\right) d x^{n+1} \\
& \quad=\left(\lambda \cdot x^{n}-\lambda \cdot y^{n}\right) \int_{\left[\lambda \cdot y^{n}, \lambda \cdot x^{n}\right]} g^{(n+1)}(t) d t \\
& \quad=\left(\lambda \cdot x^{n}-\lambda \cdot y^{n}\right)\left[\lambda \cdot y^{n}, \lambda \cdot x^{n}\right] g^{(n)}=\int_{\lambda \cdot y^{n}}^{\lambda \cdot x^{n}} g^{(n+1)}(t) d t
\end{aligned}
$$

it follows on successively reducing the integrals in (2.14) that we eventually get

$$
\int_{1} \cdots \int_{n} \int_{\lambda \cdot y^{0}}^{\lambda \cdot x} \int_{\lambda \cdot y^{1}}^{t_{1}} \int_{\lambda \cdot y^{n}}^{t_{n}} g^{(n+1)}\left(t_{n+1}\right) d t_{n+1} \cdots d t_{1} d y^{n} \cdots d y^{1} .
$$

Putting $\lambda \cdot y^{j}=u_{j}(j=0,1, \ldots, n)$ and applying (2.1), the above reduces to

$$
\int_{[1]} \cdots \int_{[n]} \int_{u_{0}}^{\lambda \cdot x} \int_{u_{1}}^{t_{1}} \cdots \int_{u_{n}}^{t_{n}} g^{(n+1)}\left(t_{n+1}\right) d t_{n+1} \cdots d t_{1} d u_{n} \cdots d u_{1}
$$

where $\int_{[j]} h=j ! \int_{\left[\lambda \cdot \rho_{j}\right]} h(j=1, \ldots, n)$. But by $(2.10)$, we see that $(2.15)$ is precisely $g(\lambda \cdot x)-G_{n}\left(g \mid \lambda \cdot \rho_{0}, \ldots, \lambda \cdot \rho_{n}\right)(\lambda \cdot x)$ which by Proposition 4 reduces to $f(x)-\mathcal{G}_{n} f(x)$ and this proves (2.13).

In order to prove $(2.11)$, we put

$$
h_{j}=D_{x-y^{0}} \prod_{\nu=1}^{j-1} D_{x^{\nu}-y^{\nu}} f, \quad j=1,2, \ldots, n,
$$

and observe that on using (2.3), we have

$$
\int_{\left[y^{j}, x^{j}\right]} D_{x^{j}-y^{j}} h_{j}\left(x^{j+1}\right) d x^{j+1}=h_{j}\left(x^{j}\right)-h_{j}\left(y^{j}\right)
$$

so that

$$
R_{j} f(x)=R_{j-1} f(x)-P_{j} f(x) .
$$


Since $R_{j} f(x)=f(x)-\mathcal{G}_{j} f(x)$, we get

$$
\mathcal{G}_{j} f(x)-\mathcal{G}_{j-1} f(x)=\rho_{j} f(x) .
$$

Relation (2.11) follows from summing this for $j=1, \ldots, n$, and recalling that $\mathcal{G}_{0} f(x)=f\left(y^{0}\right)$.

REMARK. (2.12) and (2.13) for $P_{n} f$ and $R_{n} f$, respectively, can be considerably simplified if we introduce the following notation. If $\omega^{0}, \omega^{1}, \ldots$ are elements of $\mathbf{R}^{k}$ and $f$ is sufficiently smooth, we set

$$
\begin{aligned}
T_{0}(x ; f) & =f(x) \\
T_{n}(x ; f) & \equiv T_{n}\left(x ; \omega^{1}, \ldots, \omega^{n} ; f\right) \\
& =\int_{\left[\omega^{n}, x\right]} T_{n-1}\left(x^{n-1} ; D_{x-\omega^{n}} f\right) d x^{n-1}, \quad n \geq 1 .
\end{aligned}
$$

Similarly we define

$$
\begin{aligned}
\tilde{T}_{0}(x ; f) & =\tilde{T}_{0}\left(x ; \omega^{0} ; f\right)=f\left(\omega^{0}\right), \\
\tilde{T}_{n}(x ; f) & =\tilde{T}_{n}\left(x ; \omega^{0}, \ldots, \omega^{n} ; f\right) \\
& =\int_{\left[\omega^{n}, x\right]} \tilde{T}_{n-1}\left(x^{n-1} ; D_{x-\omega^{n}} f\right) d x^{n-1}, \quad n \geq 1 .
\end{aligned}
$$

We can then rewrite (2.12) and (2.13) as follows:

$$
\begin{gathered}
\rho_{j} f(x)=\int_{1} \ldots \int_{j} \tilde{T}_{j}\left(x ; y^{j}, \ldots, y^{0} ; f\right) d y^{j} \cdots d y^{1} \quad(j=1, \ldots, n), \\
R_{n} f(x)=\int_{1} \ldots \int_{n} T_{n+1}\left(x ; y^{n}, \ldots, y^{0} ; f\right) d y^{n} \cdots d y^{1} .
\end{gathered}
$$

3. Some lemmas. Henceforth we suppose that the elements of our triangular array lie in $\mathbf{C}^{k}(k \geq 1)$, i.e., $\rho_{n}=\left\{z^{0 n}, \ldots, z^{n n}\right\} \subset \mathbf{C}^{k}, n=0,1, \ldots$ All the results of $\S 2$ hold without change if we allow $f$ to be holomorphic in the appropriate region.

For $z, \omega \in \mathbf{C}^{k}$, we write $z \cdot \omega=z_{1} \omega_{1}+\cdots+z_{k} \omega_{k}$ and $|z|=(z \cdot \bar{z})^{1 / 2}$. If $f$ is holomorphic in a region containing $\left\{z \in \mathbf{C}^{k}:|z| \leq r\right\}$, we set

$$
M_{n}(f ; r)=\sup \left\{\left|D^{\alpha} f(z)\right|:|\alpha|=n,|z|=r\right\} .
$$

LEMMA 1. If $\alpha$ is a multi-index with $|\alpha|=\nu \leq n$, we have

$$
\begin{aligned}
& D^{\alpha} T_{n}(z ; f)=T_{n-\nu}\left(z ; D^{\alpha} f\right), \\
& D^{\alpha} \tilde{T}_{n}(z ; f)=\tilde{T}_{n-\nu}\left(z ; D^{\alpha} f\right)
\end{aligned}
$$

where $T_{n}(z ; f)$ and $\tilde{T}_{n}(z ; f)$ are given by $(2.16)$ and $(2.17)$.

PROOF. It is sufficient to prove (3.2) for $\nu=1$, i.e., for $1 \leq j \leq k$, we have

$$
D_{j} T_{n}(z ; f)=T_{n-1}\left(z ; D_{j} f\right) \text {. }
$$

We shall prove this by induction on $n$. For $n=1$, we have

$$
\begin{aligned}
D_{j} T_{1}(z ; f) & =D_{j} \int_{\left[\omega^{1}, z\right]} D_{z-\omega^{1}} f\left(z^{0}\right) d z^{0}=D_{j}\left(f(z)-f\left(\omega^{1}\right)\right) \quad \text { by }(2.3) \\
& =D_{j} f(z)=T_{0}\left(z ; D_{j} f\right) .
\end{aligned}
$$


Assume that (3.4) is valid for $n-1$. Then by (3.2) we get

$$
D_{j} T_{n}(z ; f)=\frac{\partial}{\partial z_{j}} \int_{\left[\omega^{n}, z\right]} T_{n-1}\left(z^{n-1} ; D_{z-\omega^{n}} f\right) d z^{n-1}
$$

Since $T_{n}$ is a linear operator on $f$, it follows that

$$
\begin{aligned}
\int_{\left[\omega^{n}, z\right]} & T_{n-1}\left(z^{n-1} ; D_{z-\omega^{n}} f\right) d z^{n-1} \\
= & \sum_{i=1}^{k}\left(z-\omega^{n}\right)_{i} \int_{\left[\omega^{n}, z\right]} T_{n-1}\left(z^{n-1} ; D_{i} f\right) d z^{n-1} \\
= & \sum_{i=1}^{k}\left(z-\omega^{n}\right)_{i} \int_{0}^{1} T_{n-1}\left(\omega^{n}+t\left(z-\omega^{n}\right) ; D_{i} f\right) d t .
\end{aligned}
$$

We observe that

$$
\begin{aligned}
& \frac{\partial}{\partial z_{j}} \int_{0}^{1} T_{n-1}\left(\omega^{n}+t\left(z-\omega^{n}\right) ; D_{i} f\right) d t \\
& \quad=\int_{0}^{1} D_{j} T_{n-1}\left(\omega^{n}+t\left(z-\omega^{n}\right) ; D_{i} f\right) t d t \\
& \quad=\int_{0}^{1} D_{i} T_{n-1}\left(\omega^{n}+t\left(z-\omega^{n}\right) ; D_{j} f\right) t d t
\end{aligned}
$$

where the last equality follows from the inductive hypothesis.

From (3.5) and (3.6) we have, by using (3.7),

$$
\begin{aligned}
D_{j} T_{n}(z ; f)= & \int_{0}^{1} T_{n-1}\left(\omega^{n}+t\left(z-\omega^{n}\right) ; D_{j} f\right) d t \\
& +\sum_{i=1}^{k}\left(z-\omega^{n}\right)_{i} D_{i} \int_{0}^{1} T_{n-1}\left(\omega^{n}+t\left(z-\omega^{n}\right) ; D_{j} f\right) t d t \\
= & \int_{0}^{1} h(t) d t+\int_{0}^{1} t h^{\prime}(t) d t \\
= & h(1)=T_{n-1}\left(z ; D_{j} f\right)
\end{aligned}
$$

where

$$
h(t)=T_{n-1}\left(\omega^{n}+t\left(z-\omega^{n}\right) ; D_{j} f\right) .
$$

This completes the induction. Relation (3.3) follows similarly.

When $\omega^{1}=\cdots=\omega^{n}=0$ in $(2.16)$, we shall denote $T_{n}(z ; f)$ by $T_{n}^{0}(z ; f)$.

LEMMA 2. We have

$$
\left|T_{n}^{0}(z ; f)\right| \leq \frac{k^{n}|z|^{n}}{n !} M_{n}(f ;|z|)
$$


ProOF. We prove (3.8) by induction on $n$. It is clearly true for $n=0$. If (3.8) is valid for $n-1$, then

$$
\begin{aligned}
\left|T_{n}^{0}(z ; f)\right| & =\left|\int_{[0, z]} T_{n-1}^{0}\left(w ; D_{z} f\right) d w\right| \\
& =\left|\int_{[0, z]} \sum_{i=1}^{k} z_{i} T_{n-1}^{0}\left(w ; D_{i} f\right) d w\right| \\
& \leq \int_{[0, z]} \sum_{i=1}^{k}\left|z_{i}\right| \frac{k^{n-1}|w|^{n-1}}{(n-1) !} M_{n-1}\left(D_{i} f ;|w|\right) d w \\
& \leq \frac{k^{n}|z|}{(n-1) !} M_{n}(f ;|z|) \int_{[0, z]}|w|^{n-1} d w .
\end{aligned}
$$

Since

$$
\int_{[0, z]}|w|^{n-1} d w=\int_{0}^{1}|t z|^{n-1} d t=\frac{|z|^{n-1}}{n}
$$

the inequality (3.8) follows immediately.

For given $v^{0}, v^{1}, \ldots$ in $\mathbf{C}^{k}$, we define

$$
\begin{aligned}
& Q_{0, n}\left(z^{1} ; f\right)=T_{n}^{0}\left(v^{0} ; f\right) \\
& Q_{m, n}\left(z^{1} ; f\right)=\int_{\left[v^{1}, z^{1}\right]} \cdots \int_{\left[v^{m}, z^{m}\right]} T_{n}^{0}\left(v^{0} ; \prod_{i=1}^{m} D_{z^{i}-v^{i}} f\right) d z^{m+1} \cdots d z^{2} .
\end{aligned}
$$

Observe that, for $1 \leq i \leq k$,

$$
\frac{\partial}{\partial z_{i}} T_{n}^{0}\left(v^{0} ; D_{z} g\right)=\frac{\partial}{\partial z_{i}} \sum_{j=1}^{k} z_{j} T_{n}^{0}\left(v^{0} ; D_{j} g\right)=T_{n}^{0}\left(v^{0} ; D_{i} g\right)
$$

A similar computation confirms the identity

$$
\frac{\partial}{\partial z_{i}} T_{n}^{0}\left(v^{0} ; D_{z}^{p} g\right)=p T_{n}^{0}\left(v^{0} ; D_{z}^{p-1} D_{i} g\right), \quad p \geq 1
$$

This leads to

LEMMA 3. The following representation is valid for $m \geq 1$ :

$$
Q_{m, n}(z ; f)=\frac{1}{m !} T_{n}^{0}\left(v^{0} ; D_{z}^{m} f\right)-\sum_{j=0}^{m-1} \frac{1}{(m-j) !} Q_{j, n}\left(z ; D_{\nu^{j+1}}^{m-j} f\right)
$$


ProOF. We note that

$$
\begin{aligned}
\int_{\left[v^{m}, z^{m}\right]} & T_{n}^{0}\left(v^{0} ; \prod_{\nu=1}^{m} D_{z^{\nu}-v^{\nu}} f\right) d z^{m+1} \\
= & \int_{\left[v^{m}, z^{m}\right]} \sum_{i=1}^{k}\left(z^{m}-v^{m}\right)_{i} T_{n}^{0}\left(v^{0} ; \prod_{\nu=1}^{m-1} D_{z^{\nu}-v^{\nu}} D_{i} f\right) d z^{m+1} \\
= & \int_{\left[v^{m}, z^{m}\right]} \sum_{i=1}^{k}\left(z^{m}-v^{m}\right)_{i} \frac{\partial}{\partial z_{i}^{m+1}} T_{n}^{0}\left(v^{0} ; \prod_{\nu=1}^{m-1} D_{z^{\nu}-v^{\nu}} D_{z^{m+1}} f\right) d z^{m+1} \\
= & \left.T_{n}^{0}\left(v^{0} ; \prod_{\nu=1}^{m-1} D_{z^{\nu}-v^{\nu}} D_{z^{m}} f\right)-T_{n}^{0}\left(v^{0} ; \prod_{\nu=1}^{m-1} D_{z^{\nu}-v^{\nu}} D_{v^{m}} f\right)\right),
\end{aligned}
$$

where we have used (3.10) and (2.3) successively. Thus, we have

$$
\begin{aligned}
Q_{m, n}\left(z^{1} ; f\right)= & \int_{\left[v^{1}, z^{1}\right]} \cdots \int_{\left[v^{m-1}, z^{m-1}\right]} T_{n}^{0}\left(v^{0} ; \prod_{\nu=1}^{m-1} D_{z^{\nu}-v^{\nu}} D_{z^{m}} f\right) d z^{m} \cdots d z^{2} \\
& -Q_{m-1, n}\left(z^{1} ; D_{v^{m}} f\right) .
\end{aligned}
$$

Repeating this process successively we derive (3.11).

LEMMA 4. If $\left|v^{i}\right| \leq r, i=0,1, \ldots, m$, then for any $\rho>0$, there exists a number $K$ depending on $\rho$ such that

$$
\left|Q_{m, n}(z ; f)\right| \leq K \frac{(k r)^{n}}{n !}\left(\frac{k r}{\log 2}\right)^{m} M_{m+n}(f ; r)
$$

for $|z| \leq \rho$.

ProOF. Let $\left\{\alpha_{j}\right\}_{0}^{\infty}$ be a sequence of numbers in $\mathbf{C}$ given by the recursion relation

$$
\alpha_{j}=\frac{(k \rho)^{j}}{j !}+\sum_{\nu=1}^{j} \frac{(k r)^{\nu}}{\nu !} \alpha_{j-\nu}, \quad j=1,2, \ldots, \quad \alpha_{0}=1 .
$$

We shall first show that

$$
\left|Q_{m, n}(z ; f)\right| \leq C_{m, n} \alpha_{m}
$$

where $C_{m, n}=(k r)^{n} M_{m+n}(f ; r) / n$ !. It has been shown in [3, p. 42] that there is a constant $K$ depending only on $\rho$ such that

$$
\alpha_{m} \leq K(k r / \log 2)^{m}, \quad m=0,1, \ldots
$$

Inequality (3.12) follows from (3.14) and (3.15).

We shall prove (3.14) by induction on $m$ for fixed $n$. From (3.8) and (3.9) we see that (3.14) is true for $m=0$. We now suppose that it is true for $m=0,1, \ldots, p-1$. Then using (3.11) we get

$$
\left|Q_{p, n}(z ; f)\right| \leq \frac{1}{p !}\left|T_{n}^{0}\left(v^{0} ; D_{z}^{p} f\right)\right|+\sum_{j=0}^{p-1} \frac{1}{(p-j) !}\left|Q_{j, n}\left(z ; D_{v^{j+1}}^{p-j} f\right)\right| .
$$


From (3.8) we see that for $|z| \leq \rho$, we have

$$
\begin{aligned}
\left|T_{n}^{0}\left(v^{0} ; D_{z}^{p} f\right)\right| & \leq \frac{k^{n}\left|v^{0}\right|^{n}}{n !} M_{n}\left(D_{z}^{p} f ;\left|v^{0}\right|\right) \\
& \leq \frac{(k r)^{n}}{n !} k^{p}|z|^{p} M_{n+p}(f ; r) \leq C_{p, n}(k \rho)^{p} .
\end{aligned}
$$

Similarly, by the inductive hypothesis, we have

$$
\begin{aligned}
\left|Q_{j, n}\left(z ; D_{\nu^{i+1}}^{p-j} f\right)\right| & \leq \frac{(k r)^{n}}{n !} M_{j+n}\left(D_{\nu^{j+1}}^{p-j} f ; r\right) \alpha_{j} \\
& \leq \frac{(k r)^{n}}{n !}(k r)^{p-j} M_{p+n}(f ; r) \alpha_{j} \leq C_{p, n}(k r)^{p-j} \alpha_{j} .
\end{aligned}
$$

Therefore (3.16) yields

$$
\left|Q_{p, n}(z ; f)\right| \leq C_{p, n}\left[\frac{(k \rho)^{p}}{p !}+\sum_{j=1}^{p} \frac{(k r)^{j}}{j !} \alpha_{p-j}\right]=C_{p, n} \alpha_{p} \quad \text { by (3.13). }
$$

LEMMA 5. For $n \geq 1$ and given vectors $w^{1}, \ldots, w^{n}, z^{n} \in \mathbf{C}^{k}$, we have (3.17) $T_{n}\left(z^{n} ; w^{1}, \ldots, w^{n} ; f\right)$

$$
\begin{aligned}
= & T_{n}^{0}\left(z^{n} ; f\right)-T_{n}^{0}\left(w^{n} ; f\right)-\sum_{j=1}^{n-1} \int_{\left[w^{n}, z^{n}\right]} \\
& \cdots \int_{\left[w^{j+1}, z^{j+1}\right]} T_{j}^{0}\left(w^{j} ; \prod_{\nu=j+1}^{n} D_{z^{\nu}-w^{\nu}} f\right) d z^{j} \cdots d z^{n-1} .
\end{aligned}
$$

ProOF. We prove this by induction on $n$. For $n=1$, we have

$$
\begin{aligned}
T_{1}\left(z^{1} ; w^{1} ; f\right) & =\int_{\left[w^{1}, z^{1}\right]} D_{z^{1}-w^{1}} f\left(z^{2}\right) d z^{2} \\
& =\int_{\left[0, z^{1}\right]} D_{z^{1}} f\left(z^{2}\right) d z^{2}-\int_{\left[0, w^{1}\right]} D_{w^{1}} f\left(z^{2}\right) d z^{2} \\
& =T_{1}^{0}\left(z^{1} ; f\right)-T_{1}^{0}\left(w^{1} ; f\right) .
\end{aligned}
$$

We next assume the formula to be true for $n-1$. Then we have, from (2.16), (3.18)

$$
\begin{aligned}
T_{n}\left(z^{n} ; w^{1}, \ldots, w^{n} ; f\right)= & \int_{\left[w^{n}, z^{n}\right]} T_{n-1}\left(z^{n-1} ; w^{1}, \ldots, w^{n-1} ; D_{z^{n}-w^{n}} f\right) d z^{n-1} \\
= & \int_{\left[w^{n}, z^{n}\right]} T_{n-1}^{0}\left(z^{n-1} ; D_{z^{n}-w^{n}} f\right) d z^{n-1}-\sum_{j=1}^{n-1} \int_{\left[w^{n}, z^{n}\right]} \\
& \ldots \int_{\left[w^{j+1}, z^{j+1}\right]} T_{j}^{0}\left(w^{j} ; \prod_{\nu=j+1}^{n} D_{z^{\nu}-w^{\nu}} f\right) d z^{j} \cdots d z^{n-1} .
\end{aligned}
$$

By (3.2) the first term in the above is

$$
\int_{\left[w^{n}, z^{n}\right]} D_{z^{n}-w^{n}} T_{n}^{0}\left(z^{n-1} ; f\right) d z^{n-1}=T_{n}^{0}\left(z^{n} ; f\right)-T_{n}^{0}\left(w^{n} ; f\right)
$$

which together with (3.18) gives (3.17). 


\section{Estimates for $R_{n} f$ and $P_{n} f$.}

Proposition 5. Suppose $f$ is an entire function in $\mathbf{C}^{k}$. If $|z| \leq \rho$ and if $\left|x^{i j}\right| \leq r, 0 \leq i \leq j, j=0,1,2, \ldots$, then for $n=0,1,2, \ldots$, the following estimate holds:

$$
\left|R_{n} f(z)\right| \leq \frac{(k \rho)^{n+1}}{(n+1) !} M_{n+1}(f ; \rho)+C\left(\frac{k r}{\log 2}\right)^{n+1} M_{n+1}(f ; r) .
$$

Proof. We recall (2.13a) and note that $\int_{j} d y^{j}=1$, so that in order to prove (4.1) it is sufficient to show that $\left|T_{n+1}\left(z ; y^{n}, \ldots, y^{0} ; f\right)\right|$ is bounded by the expression on the right in (4.1). But by Lemma 5 and (3.9), after an obvious adjustment of notation, we have

$$
T_{n+1}\left(z ; y^{n}, \ldots, y^{0} ; f\right)=T_{n+1}^{0}(z ; f)-T_{n+1}^{0}\left(y^{0} ; f\right)-\sum_{j=1}^{n} Q_{n-j+1, j}(z ; f) .
$$

Using Lemmas 2 and 4 , we get for $|z| \leq \rho$,

$$
\begin{aligned}
& \left|T_{n+1}\left(z ; y^{n}, \ldots, y^{0} ; f\right)\right| \\
& \quad \leq \frac{(k \rho)^{n+1}}{(n+1) !} M_{n+1}(f ; \rho)+K M_{n+1}(f ; r) \sum_{j=1}^{n+1} \frac{(k r)^{j}}{j !}\left(\frac{k r}{\log 2}\right)^{n-j+1} \\
& \quad=\frac{(k \rho)^{n+1}}{(n+1) !} M_{n+1}(f ; \rho)+K\left(\frac{k r}{\log 2}\right)^{n+1} \sum_{j=1}^{n+1} \frac{(\log 2)^{j}}{j !} M_{n+1}(f ; r)
\end{aligned}
$$

which proves the result with $C<2 K$.

Proposition 6. For $j=0,1, \ldots$ let $C_{j}=\max \left\{|y|: y \in\left[\rho_{j}\right]\right\}$ and let $d_{j}=$ $\max \left\{|y-z|: y \in\left[\rho_{j+1}\right], z \in\left[\rho_{j}\right]\right\}$. For $z^{0} \in \mathbf{C}^{k}$, let

$$
D_{n, \nu}=\left[\left\{z^{0}\right\} \cup \rho_{\nu} \cup \cdots \cup \rho_{n}\right], \quad \nu=0,1, \ldots, n,
$$

and assume that $f$ is holomorphic in a region containing $D_{n, 0}$. Then for any multiindex $\alpha$ with $0 \leq|\alpha|=\nu \leq n$, we have

$$
\left|D^{\alpha} R_{n} f\left(z^{0}\right)\right| \leq \frac{\left\{\left|z^{0}\right|+k\left(c_{\nu}+d_{\nu}+\cdots+d_{n-1}\right)\right\}^{n-\nu+1}}{(n-\nu+1) !} \operatorname{Max}_{|\beta|=n+1}\left\{\left|D^{\beta} f(z)\right|, z \in D_{n, \nu}\right\}
$$

and

$$
\left|D^{\alpha} P_{n} f\left(z^{0}\right)\right| \leq \frac{\left\{\left|z^{0}\right|+k\left(c_{\nu}+d_{\nu}+\cdots+d_{n-2}\right)\right\}^{n-\nu}}{(n-\nu) !} \operatorname{Max}_{|\beta|=n}\left|\int_{n} D^{\beta} f\right| .
$$

PROOF. We introduce some notation which we shall require. We set

$$
M_{n+1, \nu}=\operatorname{Max}_{|\beta|=n+1}\left\{\left|D^{\beta} f(z)\right|: z \in D_{n, \nu}\right\}
$$

and $\|w\|=\sum_{j=1}^{k}\left|w_{j}\right|$ for any $w \in \mathbf{C}^{k}$.

We rotate the coordinates so that $z_{i}^{0}=0, i=2, \ldots, k$. We shall prove our formula for this choice of $z^{0}$ which entails no loss of generality because of Proposition 4. Take $y^{j} \in\left[\rho_{j}\right], j=0,1, \ldots, n$, and observe that

$$
T_{1}\left(z^{0} ; f\right)=\int_{\left[0, z^{0}-y^{0}\right]} D_{z^{0}-y^{0}} f\left(z^{1}+y^{0}\right) d z^{1}
$$


and more generally

$$
\begin{aligned}
T_{n+1}\left(z^{0} ; f\right)= & \int_{\left[0, z^{0}-y^{0}\right]} \int_{\left[0, z^{1}+y^{0}-y^{1}\right]} \int_{\left[0, z^{n}+y^{n-1}-y^{n}\right]} \\
& \times D_{z^{0}-y^{0}} D_{z^{1}+y^{0}-y^{1}} \cdots D_{z^{n}+y^{n-1}-y^{n}} f\left(z^{n+1}+y^{n}\right) d z^{n+1} \cdots d z^{1} .
\end{aligned}
$$

Since we have the inequality

$$
\left|D_{a^{0}} \cdots D_{a^{n}} f(z)\right| \leq \prod_{\nu=0}^{n}\left\|a^{\nu}\right\| \max _{|\beta|=n+1}\left|D^{\beta} f(z)\right|,
$$

it follows that

$$
\begin{aligned}
& \left|T_{n+1}\left(z^{0} ; f\right)\right| \leq M_{n+1,0} \int_{\left[0, z^{0}-y^{0}\right]} \cdots \\
& \qquad \int_{\left[0, z^{n}+y^{n-1}-y^{n}\right]}\left\|z^{0}-y^{0}\right\| \prod_{\nu=1}^{n}\left\|z^{\nu}+y^{\nu-1}-y^{\nu}\right\| d z^{n+1} \cdots d z^{1} .
\end{aligned}
$$

Observe that for any nonnegative function $g(s)$, we have

$$
\begin{aligned}
& \int_{\left[0, z^{\nu}+y^{\nu-1}-y^{\nu}\right]}\left\|z^{\nu}+y^{\nu-1}-y^{\nu}\right\| g\left(\left\|z^{\nu+1}\right\|\right) d z^{\nu+1} \\
& \quad=\int_{0}^{\left\|z^{\nu}+y^{\nu-1}-y^{\nu}\right\|} g(s) d s \leq \int_{0}^{\left\|z^{\nu}\right\|+\left\|y^{\nu-1}-y^{\nu}\right\|} g(s) d s
\end{aligned}
$$

and for any $A>0, a \geq 0$, we have the inequality

$$
\int_{0}^{A} \int_{0}^{t_{1}+a} g\left(t_{2}\right) d t_{2} d t_{1} \leq \int_{0}^{A+a} \int_{0}^{t_{1}} g\left(t_{2}\right) d t_{2} d t_{1} .
$$

By repeated application of the above observation in the integrals on the right side in (4.6), we arrive at the estimate

$$
\left|T_{n+1}\left(z^{0} ; f\right)\right| \leq M_{n+1,0} \int_{0}^{B} \int_{0}^{t_{1}} \cdots \int_{0}^{t_{n}} d t_{n+1} \cdots d t_{1}
$$

where

$$
B=\left\|z^{0}\right\|+\left\|y^{0}\right\|+\sum_{\nu=1}^{n}\left\|y^{\nu}-y^{\nu-1}\right\| \leq\left|z^{0}\right|+k\left(c_{0}+d_{0}+\cdots+d_{n-1}\right) .
$$

Thus

$$
\left|T_{n+1}\left(z^{0} ; f\right)\right| \leq \frac{M_{n+1,0}}{(n+1) !}\left\{\left|z^{0}\right|+k\left(c_{0}+d_{0}+\cdots+d_{n-1}\right)\right\}^{n+1} .
$$

From (3.2) we see that for $0<|\alpha|=\nu \leq n$, we have

$$
\begin{aligned}
\left|D^{\alpha} T_{n}\left(z^{0} ; f\right)\right| & =\left|T_{n-\nu}\left(z^{0} ; D^{\alpha} f\right)\right| \\
& \leq \frac{M_{n+1, \nu}}{(n-\nu+1) !}\left\{\left|z^{0}\right|+k\left(c_{\nu}+d_{\nu}+\cdots+d_{n-1}\right)\right\}^{n-\nu+1} .
\end{aligned}
$$

From (2.13a) and (4.7) we get (4.2).

In order to prove (4.3), we note that for $|\alpha|=\nu \leq n$, from (3.3) we have

$$
\left|\int_{n} \tilde{T}_{n}\left(z^{0} ; y^{n}, \ldots, y^{0} ; f\right) d y^{n}\right|=\left|\int_{n} \tilde{T}_{n-\nu}\left(z^{0} ; y^{n}, \ldots, y^{0} ; D^{\alpha} f\right) d y^{n}\right|
$$


and representing this as a multiple integral similar to (4.5) we see that it is bounded above by

$$
\begin{aligned}
& \int_{\left[0, z^{0}-y^{\nu}\right]} \cdots \int_{\left[0, z^{n-1}+y^{n-2}-y^{n-1}\right]} \mid \int_{n} D_{z^{0}-y^{\nu}} D_{z^{1}+y^{\nu}-y^{\nu+1}} \cdots \\
& D_{z^{n-1}+y^{n-2}-y^{n-1}} D^{\alpha} f\left(y^{n}\right) d y^{n} \mid d z^{n} \cdots d z^{\nu+1} \\
& \leq \max _{|\beta|=n} \int_{n}\left|D^{\beta} f\right| \int_{\left[0, z^{0}-y^{\nu}\right]} \ldots \\
& \quad \int_{\left[0, z^{n-1}+y^{n-2}-y^{n-1}\right]}\left\|z^{0}-y^{\nu}\right\| \prod_{j=\nu+1}^{n-1}\left\|z^{j}+y^{j-1}-y^{j}\right\| d z^{n} \cdots d z^{\nu+1} .
\end{aligned}
$$

Applying the same method as above and (2.12a), we get (4.3).

5. Convergence results. We now apply the estimates of $\S 4$ to prove that under various conditions on $f$ and the triangular array, $\mathcal{G}_{n} f(z) \rightarrow f(z)$ as $n \rightarrow \infty$, or equivalently, $f(z)=\sum_{j=0}^{\infty} P_{j} f(z)$. We shall prove

THEOREM 2. Suppose $\left|z^{i j}\right| \leq r, 0 \leq i \leq j, j=0,1, \ldots$ Suppose $f$ is an entire function on $\mathbf{C}^{k}(k \geq 1)$ satisfying the inequality

$$
|f(z)| \leq C \cdot \rho^{1 / 2-k-\varepsilon} \cdot 2^{\rho / k r}, \quad|z|=\rho,
$$

where $C$ is a constant and $\varepsilon>0$ is as small as we like. Then

$$
\lim _{n \rightarrow \infty} \mathcal{G}_{n} f(z)=f(z)
$$

where the convergence is uniform on bounded subsets of $\mathbf{C}^{k}$.

THEOREM 3. Let the numbers $d_{j}$ be defined as in Proposition 6. Suppose $\sum_{j=0}^{\infty} d_{j}$ converges and that

$$
z^{0}=\lim _{\substack{0 \leq j \leq n \\ n \rightarrow \infty}} z^{j n}
$$

If $f$ is holomorphic in the region $B_{R}=\left\{z \in \mathbf{C}^{k}, k \geq 1:\left|z-z^{0}\right| \leq R\right\}$ and if $B_{R}$ contains the points $z^{j n}, 0 \leq j \leq n, n=0,1, \ldots$, then (5.2) holds, where the convergence is uniform on compact subsets of $B_{R}$.

If $\sum_{j=0}^{\infty} d_{j}$ is not convergent, we have

THEOREM 4. With the numbers $d_{j}$ defined as in Theorem 3 let

$$
N(r)=\min \left\{n: k\left(d_{0}+\cdots+d_{n-1}\right)>r\right\} .
$$

Suppose $f$ is an entire function and suppose there are numbers $\theta, \lambda$ with $0<\theta<$ $1 / 2,0<\lambda<\log \left(\theta^{-1}-1\right)$, such that for all large enough $r$, we have

$$
\max \{|f(z)|:|z|=r\}<\exp \{\lambda N(\theta r)\} .
$$

Then (5.2) holds, where the convergence is uniform on bounded subsets of $\mathbf{C}^{k}$.

The basic tool in the proofs will be the Cauchy-Szegö kernel for the ball [6, Chapter 1]:

$$
f(z)=\frac{(k-1) ! R}{2 \pi^{k}} \int_{|\omega|=R} \frac{f(\omega) d \sigma(\omega)}{\left(R^{2}-z \cdot \bar{\omega}\right)^{k}}, \quad|z|<R
$$


where $f$ is holomorphic in a region containing $\{z:|z| \leq R\}$ and $d \sigma(\omega)$ denotes surface measure.

PROOF OF THEOREM 2. For any $s>0$, choose $z^{0}$ and a multi-index $\alpha$ with $|\alpha|=n+1$ such that $\left|D^{\alpha} f\left(z^{0}\right)\right|=M_{n+1}(f ; s)$. From (5.5) we have, for any $R>s$,

$$
\left|D^{\alpha} f\left(z^{0}\right)\right|=\frac{R(k+n) !}{2 \pi^{k}}\left|\int_{|\omega|=R} \frac{\bar{\omega}^{\alpha} f(\omega) d \sigma(\omega)}{\left(R^{2}-z^{0} \cdot \bar{\omega}\right)^{k+n+1}}\right|,
$$

whence for some constant $C_{1}$ we have

$$
M_{n+1}(f ; s) \leq C_{1} \frac{(k+n) ! M_{0}(f ; R)}{R^{n+1}(1-s / R)^{k+n+1}} .
$$

For any $\rho>0$, we take $s=\rho$ and $R>(k+1) \rho$ in (5.6) which shows that

$$
\frac{(k \rho)^{n+1}}{(n+1) !} M_{n+1}(f ; \rho) \rightarrow 0 \text { as } n \rightarrow \infty .
$$

Moreover, from (5.1) and (5.6) for $R>r$ we have

$$
M_{n+1}(f ; r)<C_{2} \frac{(k+n) !}{(R-r)^{n+1}} R^{1 / 2-k-\varepsilon} \cdot 2^{R / k r} .
$$

Putting $R=r+(n+k) k r / \log 2$ in the above inequality, we get

$$
M_{n+1}(f ; r)<C_{3} \frac{(k+n) !(n+k)^{1 / 2-k-\varepsilon}}{(n+k)^{n+1}} e^{n+k}\left(\frac{\log 2}{k r}\right)^{n+1}
$$

which by Stirling's formula yields

$$
M_{n+1}(f ; r)<C_{3}(k+n)^{-\varepsilon}\left(\frac{\log 2}{k r}\right)^{n+1} .
$$

The result now follows from (5.7), (5.8) and (4.1).

PROOF OF THEOREM 3. On making a suitable translation and using Proposition 4 , we may choose $z^{0}=0$ without loss of generality. The proof will be divided into two parts (a) and (b). In (a) we shall show that

$$
\sum_{\nu=0}^{\infty} P_{\nu} f(z)=f_{1}(z)
$$

where $f_{1}(z)$ is some function holomorphic in $B_{R}$ and that the convergence is uniform on compact subsets of $B_{R}$. In (b) we show that $f_{1}(z)=f(z)$.

(a) We shall use formula (4.3) and to this effect we shall need a bound on $\left|\int_{n} D^{\beta} f\right|=n !\left|\int_{\left[\rho_{n}\right]} D^{\beta} f\right|$ with $|\beta|=n$. Take any $R_{1}, R_{2}$ with $0<R_{1}<R_{2}<R$ and for a given $\varepsilon>0$, choose $N$ so large that $\sum_{j=N}^{\infty} d_{j}<\varepsilon$ and $c_{n}<\varepsilon$ for $n \geq N$, where $c_{n}=\max \left\{|z|: z \in\left[\rho_{n}\right]\right\}$. From (5.5), we have

$$
\int_{\left[\rho_{n}\right]} D^{\beta} f(z) d z=\frac{(k-1) ! R_{2}}{2 \pi^{k}} \int_{|\omega|=R_{2}} f(\omega) \int_{\left[\rho_{n}\right]} D^{\beta}\left(R_{2}^{2}-z \cdot \bar{\omega}\right)^{-k} d z d \sigma(\omega)
$$

where $|\beta|=n$. Putting $g(t)=t^{-k}$ and $h_{j}=R_{2}^{2}-z^{j n} \cdot \bar{\omega}(j=0,1, \ldots, n)$, we have

$$
\begin{aligned}
\int_{\left[\rho_{n}\right]} D^{\beta}\left(R_{2}^{2}-z \cdot \bar{\omega}\right)^{-k} d z & =(-\bar{\omega})^{\beta} \int_{\left[h_{0}, \ldots, h_{n}\right]} g^{(n)}(t) d t \\
& =(-\bar{\omega})^{\beta}\left[h_{0}, \ldots, h_{n}\right] g .
\end{aligned}
$$


It is easy to verify (see also $[1$, Lemma 1.10, p. 75$]$ ) that

$$
\left[h_{0}, \ldots, h_{n}\right] g=\frac{(-1)^{n}}{h_{0} \cdots h_{n}} \sum_{|\gamma|=k-1} 1 / h^{\gamma}
$$

where $h=\left(h_{0}, \ldots, h_{n}\right)$ and $\gamma=\left(\gamma_{0}, \ldots, \gamma_{n}\right)$. From (5.10), (5.11) and (5.12), we have for $n \geq N$,

$$
\begin{aligned}
\left|\int_{n} D^{\beta} f\right| & \leq \frac{n !(k-1) ! R_{2}}{2 \pi^{k}} \cdot \frac{M_{0}\left(f ; R_{2}\right) K_{1} R_{2}^{2 k-1} R_{2}^{n}}{\left(R_{2}^{2}-R_{2} \varepsilon\right)^{n+1}}\left(\begin{array}{c}
n+k-1 \\
k-1
\end{array}\right) \frac{1}{\left(R_{2}^{2}-R_{2} \varepsilon\right)^{k-1}} \\
& \leq K_{2} \cdot \frac{n ! n^{k-1}}{\left(R_{2}-\varepsilon\right)^{n}}
\end{aligned}
$$

where $K_{1}, K_{2}$ are constants independent of $n$. We now use (4.3) with $|\alpha|=N,|z| \leq$ $R_{1}$ and obtain

$$
\left|D^{\alpha} P_{n} f(z)\right| \leq \frac{\left(R_{1}+2 k \varepsilon\right)^{n-N}}{(n-N) !} \frac{K_{2} n ! n^{k-1}}{\left(R_{2}-\varepsilon\right)^{n}} \leq K_{3}^{N+k-1}\left(\frac{R_{1}+2 k \varepsilon}{R_{2}-\varepsilon}\right)^{n} .
$$

Choosing $\varepsilon$ so small that $\left(R_{1}+2 k \varepsilon\right) /\left(R_{2}-\varepsilon\right)<1$, we see that $\sum_{n=N}^{\infty} D^{\alpha} P_{n} f(z)$ converges uniformly for $|z| \leq R_{1}$. We can then integrate $N$ times to get the uniform convergence of $\sum_{\nu=0}^{\infty} P_{\nu} f(z)$ in $|z| \leq R_{1}$. Since $R_{1}<R$ was arbitrary this gives (5.9).

(b) For a multiple index $\beta$ with $|\beta|=n+1$ and for $|z|<\varepsilon$, we have from (5.5)

$$
\begin{aligned}
\left|D^{\beta} f(z)\right| & =\frac{(k+n) ! R_{2}}{2 \pi^{k}}\left|\int_{|\omega|=R_{2}} \frac{(-\bar{\omega})^{\beta} f(\omega) d \sigma(\omega)}{\left(R_{2}^{2}-z \cdot \bar{\omega}\right)^{k+n+1}}\right| \\
& \leq \frac{K_{4}(k+n) !}{\left(R_{2}-\varepsilon\right)^{n}} \quad\left(K_{4} \text { independent of } n\right) .
\end{aligned}
$$

Applying (4.2), we have for $|\alpha|=N \leq n$

$$
\begin{aligned}
\left|D^{\alpha} R_{n} f(z)\right| & \leq \frac{((2 k+1) \varepsilon)^{n-N+1}}{(n-N+1) !} K_{4} \cdot \frac{(k+n) !}{\left(R_{2}-\varepsilon\right)^{n}} \\
& \leq K_{5} n^{k+N-1}\left(\frac{2 k+1}{R_{2}-\varepsilon} \varepsilon\right)^{n} .
\end{aligned}
$$

If we choose $\varepsilon$ such that $(2 k+1) \varepsilon /\left(R_{2}-\varepsilon\right)<1$, we see that $D^{\alpha} R_{n} f(z) \rightarrow 0$ uniformly in $|z| \leq \varepsilon$ which is equivalent to saying that $D^{\alpha} \mathcal{G}_{n} f(z) \rightarrow D^{\alpha} f(z)$.

From (5.9) we know that $D^{\alpha} \mathcal{G}_{n} f(z) \rightarrow D^{\alpha} f_{1}(z)$. Thus $D^{\alpha} f(z)=D^{\alpha} f_{1}(z)$ in $|z|<\varepsilon$ and hence for $z \in B_{R}$ for all $\alpha$ with $|\alpha|=N$. Hence $f(z)-f_{1}(z)$ is a polynomial of degree $\leq N-1$. Since

$$
\int_{\left[\rho_{\nu}\right]} D^{\beta} f=\int_{\left[\rho_{\nu}\right]} D^{\beta} \mathcal{G}_{N}=\int_{\left[\rho_{\nu}\right]} D^{\beta} f_{1}, \quad|\beta|=\nu, \quad \nu=0,1, \ldots, N-1,
$$

it follows that $f(z)-f_{1}(z)=0$, which completes the proof of Theorem 3 .

PROOF OF THEOREM 4. For a fixed $\rho>0$, set

$$
\mu_{n}=\rho+k\left(\left|z^{00}\right|+d_{0}+\cdots+d_{n-1}\right) .
$$


Choose $\eta$ with $0<\eta<\theta^{-1}$. Since $\sum_{j=0}^{\infty} d_{j}$ diverges, it follows that for large enough $n$ we have

$$
\theta \eta \mu_{n}<k\left(d_{0}+\cdots+d_{n-1}\right) .
$$

From (5.15) and the definition of $N(r)$, we have $N\left(\theta \eta \mu_{n}\right) \leq n$. Hence from (5.4), we obtain

$$
M_{0}\left(f ; \eta \mu_{n}\right)<\exp \left\{\lambda N\left(\theta \eta \mu_{n}\right)\right\} \leq \exp (\lambda n) .
$$

If $|z|<\rho$ we use (4.2) with $\nu=0$ and obtain

$$
\left|R_{n} f(z)\right| \leq \frac{\mu_{n}^{n+1}}{(n+1) !} M_{n+1}\left(f ; \mu_{n}\right), \quad|z|<\rho .
$$

If we also require $\eta>1$, then from (5.5) we have for $|z|=\mu_{n}$ and $|\alpha|=n+1$,

$$
\begin{aligned}
\left|D^{\alpha} f(z)\right| & =\frac{(k+n) ! \eta \mu_{n}}{2 \pi^{k}}\left|\int_{|\omega|=\eta \mu_{n}} \frac{\bar{\omega}^{\alpha} f(\omega) d \sigma(\omega)}{\left(\eta^{2} \mu_{n}^{2}-z \cdot \bar{\omega}\right)^{k+n+1}}\right| \\
& \leq K_{6} \frac{(k+n) ! M_{0}\left(f ; \eta \mu_{n}\right)}{\mu_{n}^{n+1}(\eta-1)^{k+n+1}} \leq \frac{K_{6}(k+n) ! \exp \lambda n}{\mu_{n}^{n+1}(\eta-1)^{k+n+1}} \quad \text { by (5.16). }
\end{aligned}
$$

From (5.17) and (5.18), we obtain

$$
\left|R_{n} f(z)\right| \leq K_{6} \frac{(k+n) ! \exp \lambda n}{(n+1) !(\eta-1)^{k+n+1}}<K_{7} \cdot n^{k} \exp \{\lambda-\log (\eta-1)\} n .
$$

Choosing $\eta$ so that $\log (\eta-1)>\lambda$, we get the convergence of $R_{n} f(z)$ to zero uniformly for $|z| \leq \rho$.

\section{REFERENCES}

1. T. Bloom, Kergin interpolation of entire functions on $C^{n}$, Duke Math. J. 48 (1981), 69-83.

2. A. S. Cavaretta, Jr., C. A. Micchelli and A. Sharma, Multivariate interpolation and the Radon transform, Math. Z. 174 (1980), 263-279.

3. A. O. Gelfond, Calcul des differences finis, Dunod, Paris, 1963.

4. P. Kergin, A natural interpolation of $C^{k}$ functions, J. Approx. Theory 29 (1980), 278-293.

5. C. A. Micchelli, A constructive approach to Kergin interpolation in $\mathbf{R}^{k}$ : Multivariate $B$-splines and Lagrange interpolation, Rocky Mountain J. Math. 10 (1980), 485-497.

6. E. Stein, Boundary values of holomorphic functions of several complex variables, Math. Notes, Princeton Univ. Press, Princeton, N.J.

Department of Mathematics, University of Dundee, Dundee, Scotland

Department of Mathematics, University of Alberta, Edmonton, Alberta T6G 2G1, CANADA

Current address: Department of Mathematics, Kent State University, Kent, Ohio 44242 